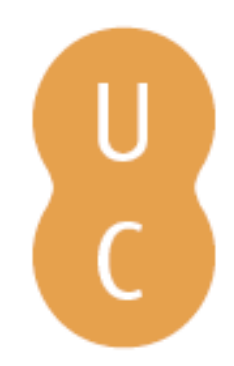

\title{
nombalina
}

\section{Improving risk matrices using the MACBETH approach for multicriteria value measurement}
Autor(es):
Costa, Carlos A. Bana e; Lopes, Diana F.; Oliveira, Mónica D.
Publicado por: Imprensa da Universidade de Coimbra; Faculdade de Ciências e
persistente:
Mecnologica
DOI:
URI:http://hdl.handle.net/10316.2/33345
DOI:http://dx.doi.org/10.14195/978-972-8954-42-0_27
Accessed : $\quad$ 26-Apr-2023 09:24:10

A navegação consulta e descarregamento dos títulos inseridos nas Bibliotecas Digitais UC Digitalis, UC Pombalina e UC Impactum, pressupõem a aceitação plena e sem reservas dos Termos e Condições de Uso destas Bibliotecas Digitais, disponíveis em https://digitalis.uc.pt/pt-pt/termos.

Conforme exposto nos referidos Termos e Condições de Uso, o descarregamento de títulos de acesso restrito requer uma licença válida de autorização devendo o utilizador aceder ao(s) documento(s) a partir de um endereço de IP da instituição detentora da supramencionada licença.

Ao utilizador é apenas permitido o descarregamento para uso pessoal, pelo que o emprego do(s) título(s) descarregado(s) para outro fim, designadamente comercial, carece de autorização do respetivo autor ou editor da obra.

Na medida em que todas as obras da UC Digitalis se encontram protegidas pelo Código do Direito de Autor e Direitos Conexos e demais legislação aplicável, toda a cópia, parcial ou total, deste documento, nos casos em que é legalmente admitida, deverá conter ou fazer-se acompanhar por este aviso.

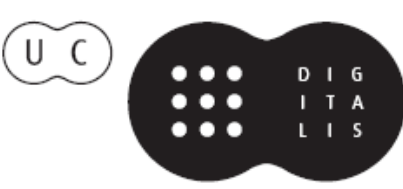




\title{
Improving risk matrices using the MACBETH approach for multicriteria value measurement
}

\author{
Carlos A. Bana e Costa ${ }^{1}$; Diana F. Lopes ${ }^{2}$; Mónica D. Oliveira ${ }^{3}$ \\ 1'carlosbana@tecnico.ulisboa.pt; ${ }^{2}$ diana.lopes@tecnico.ulisboa.pt; ${ }^{3}$ monica.oliveira@tecnico.ulisboa.pt \\ CEG-IST, Centre for Management Studies of Instituto Superior Técnico, Universidade de Lisboa, \\ Avenida Rovisco Pais, 1049-001 Lisbon, Portugal
}

\begin{abstract}
Risk matrices (RMs) have been recommended by many organizations to evaluate and mitigate risks. This study aims at improving the design and the deployment of RMs, avoiding theoretical problems of traditional RMs and inconsistent risk ratings, following Multicriteria and Portfolio Decision Analysis. In particular, the MACBETH approach is used to build quantitative evaluation models from qualitative value judgments. A new RMs' modeling framework is proposed, which includes: (1) the construction of a multicriteria additive value model applying MACBETH to assess risk impacts; (2) the innovative use of MACBETH to derive subjective probabilities; (3) the transformation of a RM into a Value Risk Matrix (VRM); (4) the definition of multicriteria assignment procedures, applied to classify risks from the VRM by severity; (5) and the use of MACBETH's resource allocation to prioritize risk mitigation actions and analyze portfolios that offer the best value for money for different budgeting and contextual constraints.
\end{abstract}

Keywords- Risk evaluation, Multiple Criteria and Portfolio Decision Analysis, MACBETH.

\section{INTRODUCTION}

Organizations have been looking for processes to prevent risks that threaten the goals of their projects. In particular, risk managers are increasingly recognizing the need to determine the relative significance of different sources of risk, being this required to guide a cost effective management of risks (Chapman et al., 2007; Bertsch, 2008). This topic is even more critical in the context of tighter resource constraints (Dillon et al., 2003).

Risk matrices (i.e. tables mapping 'frequency' and 'severity' ratings to corresponding risk priority levels) have been recommended by international organizations - such as by the Project Management Institute (Project Management Institute, 2004) - to assess risk. Their use is rather appealing because they are easy to handle, demand for limited expertise, have a straightforward interpretation and allow for performing a quick analysis. Their incorporation in software packages has spread its use. Nevertheless, available studies indicate that the use of risk matrices (RMs) might generate inconsistencies in risk management and RMs do not respect important theoretical properties (Cox Jr., 2008). For example, RMs replicate non-compensatory decision rules that may not reflect the risk preferences of decision-makers (DM) across risk impacts in different criteria nor the multidimensional and compensatory nature of risk impacts. They do not respect the principle of translation invariance and, above all, RMs can lead to a suboptimal allocation of resources. Despite these problems, few studies have researched improvements to RMs.

This study proposes multiple criteria decision analysis (MCDA) methods, based on the Measuring Attractiveness by a Categorical Based Evaluation Technique (MACBETH) approach, to improve the design and the deployment of RMs to prioritize risks and interventions to reduce risk in the context of scarce resources. MCDA provides a set of methods with theoretical foundations and that can be used to overcome key weaknesses of RMs. In particular, MCDA methods and the MACBETH approach allow for accounting for the multiple dimensions of risk impacts (e.g. on budget, quality, delivery time, etc). Also they allow for accounting for quantitative and qualitative (objective) information of risks and for the subjective preferences of DMs while respecting the principle of translation invariance and promoting an optimal allocation of scarce resources

In this article we briefly describe how MCDA and MACBETH can improve the design of RMs, by following a modeling approach based on:

(1) a multi-criteria additive value model applying MACBETH to assess risk impacts;

(2) the modeling of probabilities using subjective and qualitative preference information and MACBETH;

(3) the use of multicriteria classification procedures to classify risks by severity;

(4) the transformation of a risk matrix into a Value Risk Matrix (VRM) that uses probabilities, multicriteria value and risk classifications from (1) to (3);

(5) the use of the recent portfolio decision analysis component of the M-MACBETH Decision Support System (DSS) that combined with VRM can derive the most effective set of interventions to reduce risk, while taking into account of costs and other constraints;

(6) modeling uncertainty in (1) to (2) so as to represent uncertainty in (4) and to perform robustness analysis in (5).

We are developing the proposed methods, as well as we are applying them to real case studies in risk management, including to the evaluation of health and safety risks. The proposed methods follow a socio-technical approach, with the technical component above described, and with the social component being defined by the use of participatory methods to build a compromise and confidence between stakeholders 
who participate in risk management. Nevertheless, in this paper we do not cover the social aspects related with the design and use of RMs (Phillips et al., 2007). The application of the proposed methods is supported by decision support systems including several components of the M-MACBETH DSS (Bana Consulting, 2005). We believe that the results of applying the proposed methods to improve RMs will correct existing problems in RMs and thus improve risk management procedures.

\section{LITERATURE REVIEW}

Risk management relates to all sources of risk that can affect an organization in terms of its goals and vision [1]. To compare and assess risks and to define risk management policies, RMs have been recommended by international organizations, such as United States Department of Defense and the National Patient Safety Agency from the United Kingdom, and are widely used as a framework for practical risk analysis. Appearing in literature under different designations - probability impact table [2], probability and impact matrix [3], probability impact diagram [4], probability impact grid [5] and risk maps [6] - risk matrices are tables (or plots) that have categories of 'probability', 'likelihood' or 'frequency' for its rows (or columns) and categories of 'severity', 'impact' or 'consequences' for its columns (or rows, respectively) [7]. Typically, in each RM, a recommended level of risk, urgency, priority or management action is presented in each row-column pair; risks categorized with higher priority are given a higher managerial attention for treatment and mitigation; and each RM can be used to describe the impact of a risk in a single dimension or in multiple dimensions (in this case a measure of global impact is represented in the matrix).

For example, the Health Service Executive of Ireland is using the matrix presented in table I and has defined three risk categories by applying that expected risk measure upon two 5level rating scales, as follows [8]: "The high risks are scored between 15 and 25 and are colored Red"; "Medium risks are scored between 6 and 12 and are colored Amber"; "Low risks are scored between 1 and 5 and are colored Green". In fact, the higher the 'risk rating', the more 'severe' the risk classified, having a higher score; and a type of traffic light system is used to break risks into groups requiring different response strategies. Similar systems have been used by organizations such as the US Department of Defense [9] and the Construction and Commission Department at ALSTOM [4].

TABLE I. EXAMPLE OF A QUANTITATIVE RISK MATRIX (ADAPTED FROM (QUALITY AND PATIENT SAFETY DIRECTORATE, 2011)), WITH THREE RISK CATEGORIES

\begin{tabular}{|c|c|c|c|c|c|}
\cline { 2 - 6 } \multicolumn{1}{c|}{} & Negligible (1) & Minor (2) & Moderate (3) & Major (4) & Dxtreme (5) \\
\hline Almost Certain (5) & $\mathbf{5}$ & $\mathbf{1 0}$ & 15 & 20 & 25 \\
\hline Likely (4) & $\mathbf{4}$ & $\mathbf{8}$ & $\mathbf{1 2}$ & 16 & 20 \\
\hline Possible (3) & $\mathbf{3}$ & $\mathbf{6}$ & $\mathbf{9}$ & $\mathbf{1 2}$ & 15 \\
\hline Unilikely (2) & $\mathbf{2}$ & 4 & 6 & 8 & 10 \\
\hline Rare/Remote (1) & $\mathbf{1}$ & $\mathbf{2}$ & $\mathbf{3}$ & $\mathbf{4}$ & $\mathbf{5}$ \\
\hline
\end{tabular}

The use of RMs is rather appealing because their use is intuitive and demands for limited expertise. RMs have a straightforward interpretation, are perceived as transparent tools and quickly provide a rough discrete (ordered categorical) approximation to a more detailed underlying quantitative relation [7]. Several software packages make use of RMs - for example the Active Risk Manager (ARM) [4], the MITRE's risk matrix tool [10] and the SAP software [11] for program risk management - enabling their use in different risk management contexts. By providing a clear framework for systematic review of risks, the use of RMs enables organizations to prepare convenient documentation for risk management and provides an opportunity for stakeholders participating in the process of building RMs [12].

Nevertheless, RMs should not be seen as a quantitative risk analysis method [2]. Their use should be aware that they do not respect important theoretical properties and their utilization might generate inconsistencies in risk management, namely:

a) RMs replicate non-compensatory decision rules that may not reflect the risk preferences of real DMs across risk impacts in different criteria [12];

b) RMs violate the principle of translation invariance [13];

c) RMs provide an approximation that can only be satisfactory if certain conditions are satisfied (e.g., in terms of preferences). However, as the risk attitudes of the RM builders are seldom documented, it can be impossible to determine how consequence severity classifications should be changed when someone else views or uses the matrix [Co09];

d) RMs use a discrete value function consisting on bands of impact and probability instead of a continuous value function. A discrete value function increases only by jump discontinuities and the border points where jumps occur are not always discussed. Also, RMs have often poor resolution, meaning that they can correctly and unambiguously compare only a small fraction of risks [Co09];

e) And above all, the use of RMs can lead to suboptimal resource allocation (for example, risks with high ratings typically receive higher priority for treatment and mitigation, which might be inadequate [Co08]), and there has been little rigorous empirical or theoretical study on how well RMs succeed in improving risk management decisions [Co09].

Other issues have been pointed out in literature, such as it is hard to dynamically use RMs, and difficult to incorporate and convey uncertainty in RMs.

Despite an increased interest from academia and organizations in risk management in recent years $[14,15]$ and some recent studies attempting to improve RMs, such as developing the fuzzy risk matrix [16], the RM with Borda method [9] and mathematical operations in inputs of RMs [9], major limitations of risks matrices are still unsolved [9] and further theoretical and applied research is needed [7]. 


\section{METHODOLOGICAL FRAMEWORK}

This study discusses how multiple criteria decision analysis (MCDA) methods, based on the MACBETH approach, can be used to improve the design and the deployment of RMs in risk management, and to build VRMs. We name this the IRIS approach. MCDA provides a set of methods with theoretical foundations [17-19] that can be used to evaluate and order risks and help to overcome the weaknesses of RMs identified in the literature. First of all, MCDA methods allow for accounting for the multidimensional impacts (for example, on budget, quality and delivery time - the triple constraint in project management). Secondly, MCDA methods allow for accounting for various levels of quantitative and qualitative information of risks, as well as for subjective preferences of DMs, which are key features in risk evaluation [7]. Thirdly, MCDA methods can be used to change RMs so as to respect the principle of translation invariance by assisting in the quantification of the impact of risks and of probabilities. Last, and above all, MCDA methods - in particular methods from portfolio decision analysis - can help to overcome the problem of suboptimal resource allocation through the use of multicriteria resource allocation models to select risk interventions. As mentioned above, we propose the use of MACBETH, that is an interactive multicriteria decision analysis approach used to build a quantitative (numerical) value model based on nonnumerical (qualitative) pairwise comparison judgments [2023]. MACBETH approach requires qualitative pairwise comparison judgments of difference in attractiveness (value), therefore involving only two elements in each judgment, to help an individual or group of decision makers to score options on each criterion and to weight criteria [24]. Previous studies have shown that MACBETH provides a simple and transparent approach in modelling complex multidimensional problems, and hence its wide applicability in MCDA (see for example $[23,25,26])$

The IRIS approach makes use of MACBETH in several of the activities presented in grey in Fig. 1, which defines our proposed modeling approach:

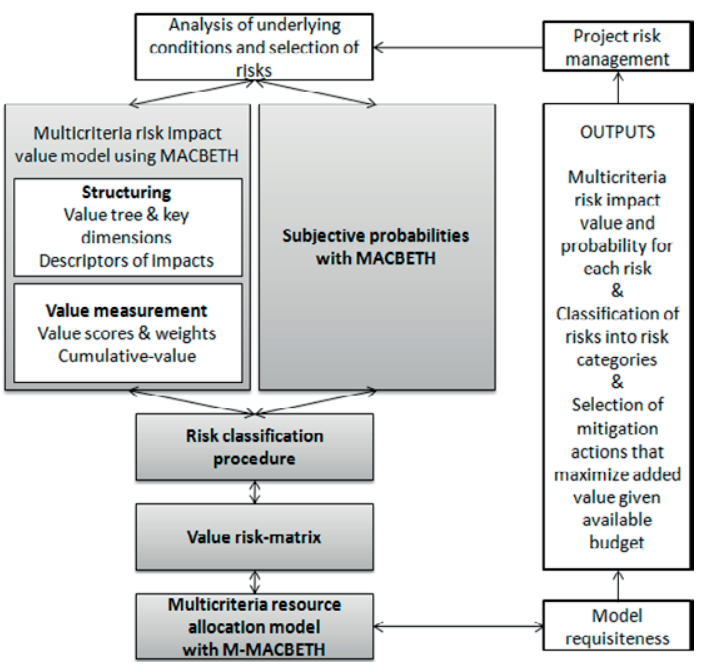

\section{IRIS ACTIVITIES}

\section{A. Multicriteria Risk Impact Value Model}

This section proposes the development of a multi-criteria additive value model applying the MACBETH approach [27] to measure risk impact value. Multicriteria value models allow for understanding how risk impacts are not important by themselves, but by the way they might contribute for creating or destroying value in an organization; and for analyzing impacts (and also probabilities) in a continuous scale (so as to avoid discontinuity problems), even if impacts might be qualitatively measured.

The development of a multi-criteria (hierarchical) additive value model applying MACBETH to measure risk impacts has already been tested in a real case study at ALSTOM Power (Switzerland) in the context of construction-related risks [4]. Results from this application have shown key advantages of using multicriteria value models to evaluate risk impacts in the context of RMs. We illustrate how to apply the proposed methods with application to the ALSTOM case.

Following Fig. 1, the development of multicriteria risk impact models involves structuring and evaluation activities [23]. Fig. 2 shows the value tree built in decision conferences at ALSTOM [4]. There are six evaluation criteria (in italics) considered to be exhaustive, non-redundant and additively independent. To appraise the extent to which different risk events can impact on the six criteria, an attribute or descriptor of impacts was associated to each of them [28] [29]. Table II shows the descriptor of impacts for the Schedule criterion.

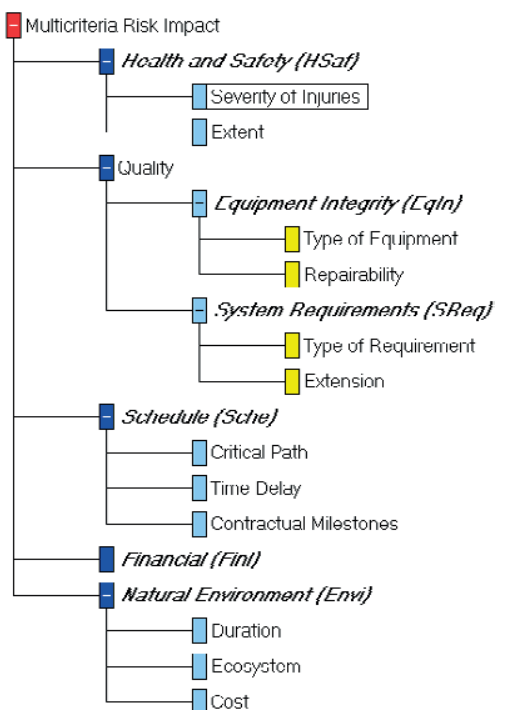

Fig. 2. A Value tree in the M-MACBETH DSS (adapted from (Figueiredo et al., 2009)), being all the criteria (key dimensions) in italics and their abbreviations in brackets.

Fig. 1. Activities and outputs. 
TABLE II. CONSTRUCTED DESCRIPTOR FOR THE SCHEDULE CRITERION (ADAPTED FROM (FIGUEIREDO, 2008))

\begin{tabular}{|c|l|}
\hline Impact levels & \multicolumn{1}{|c|}{ Description } \\
\hline No effect & $\begin{array}{l}\text { No impact on the schedule of the project (depicting no } \\
\text { changes to the Status Quo) }\end{array}$ \\
\hline Effect TD & $\begin{array}{l}\text { Impact on the schedule of the project, but not affecting } \\
\text { the internal critical path }\end{array}$ \\
\hline+ Small CP & $\begin{array}{l}\text { Impact on the internal critical path less than 10 days, } \\
\text { but no impact on contractual milestones }\end{array}$ \\
\hline+ High CP & $\begin{array}{l}\text { Impact on the critical path equal or higher than 10 } \\
\text { days, but no impact on contractual milestones }\end{array}$ \\
\hline+ CM & $\begin{array}{l}\text { Impact on critical path and on contractual milestones } \\
\text { (implying the payment of Late Deliveries) }\end{array}$ \\
\hline
\end{tabular}

Evaluation activities involve creating a model of intracriteria preferences that evaluate the impacts of different risks for each criterion, through the use of value scales or value functions (see Fig. 3), as well as the elicitation of relative weights for the criteria (see Fig. 4). These steps are required for assessing risk impacts on a common value scale. Value functions and weights require DM's judgments, being the subjective component of the multicriteria value model, and with the MACBETH approach assisting in this task. For building value functions, the MACBETH questioning protocol consists in asking the DM to qualitatively judge the difference in attractiveness between impact levels, two at a time, based on seven semantic categories: "is there no difference, or is the difference very weak, weak, moderate, strong, very strong, or extreme?" (Bana e Costa et al., 2012). During this questioning protocol, a matrix with the categorical judgments of the DM is populated (see Fig. 3 (left)). Each time a qualitative judgment is introduced in the matrix, the M-MACBETH DSS verifies its consistency and offers suggestions to solve eventual inconsistencies (Bana e Costa et al., 2008). After the consistency verification, the software derives, by mathematical programming, an interval numerical scale which has to be analyzed and validated by the DM (Bana e Costa et al., 2012) - see Fig. 3 (right). For details on the application of the MACBETH approach to build value functions and weights, consult (Bana e Costa et al., 1999; Carnero, 2006; Bana e Costa et al., 2008; Joerin et al., 2010; Barin et al., 2012).

It is worthwhile to note that the use of the recent version of M-MACBETH DSS makes available a hierarchical version of M-MACBETH, which allows for assigning weights hierarchically, as in Fig. 5. In this case, weighting is not only used to define weights at the criteria level, but also for areas which include criteria by type of concern - following Fig. 5, MACBETH allows (see green circles): (a) to weight all the criteria (similarly to Fig. 4); (b) to weight the children criteria of an area; and to weight criteria and area at same time by using (c) the information of all the criteria including all children criteria of the area or (d) the information of one children criterion of the area and all the remainder criteria.

\begin{tabular}{|c|c|c|c|c|c|c|c|c|}
\hline \multicolumn{6}{|c|}{ Schedule (Sche) } & \multicolumn{2}{|c|}{ Schedule (Sche) } & Es \\
\hline 田 & $+\mathrm{CM}$ & + High CP & + Small CP & Effect TD & No effect & \multirow[b]{3}{*}{58.99} & \multirow[t]{2}{*}{$+\mathrm{CM}$} & \multirow[t]{2}{*}{100} \\
\hline$+\mathrm{CM}$ & no & extreme & extreme & extreme & extreme & & & \\
\hline+ High CP & & no & mod-strg & strong & positive & & -+ High CP & 53 \\
\hline + Small CP & & & no & moderate & positive & & -+ Small CP & 35 \\
\hline \begin{tabular}{|l|} 
Effect ID \\
No effect
\end{tabular} & & & & no & $\begin{array}{l}\text { weak-extr } \\
\text { no }\end{array}$ & 35.01 & Effect TD & 18 \\
\hline \multicolumn{6}{|c|}{ Consistent judgements } & & No effect & 0 \\
\hline
\end{tabular}

Fig. 3. MACBETH matrix of judgments for the Schedule criterion (left) and the corresponding value scale (right) - adapted from (Figueiredo, 2008). The judgments "weak-ext" and "mod-strg" are abbreviations of "weak or extreme", "moderate or strong", respectively, and depict cases of differences in opinion or hesitation among ALSTOM risk managers (DM).

\begin{tabular}{|c|c|c|c|c|c|c|c|c|c|}
\hline \multicolumn{7}{|c|}{ Weighting (Multicriteria Risk Impact) } & $\mathbb{3}$ & Weighting (Multicri... & $\mathbb{X}$ \\
\hline 圆 & [HSaf] & [Envi] & [Finl ] & [SReq] & [Sche] & {$[$ Eqln ] } & [all SQ] & $-[$ HSaf] & 1.00 \\
\hline [HSaf] & $\mathrm{nn}$ & strn-vstr & positive & positive & positive & positive & extreme & $-[$ Envi] & 0.81 \\
\hline [Envi] & & no & strong & positive & positive & positive & vstrq-extr & & \\
\hline [Finl] & & & no & strong & positive & positive & vstrg-extr & \begin{tabular}{|l|} 
[Finl ] \\
\end{tabular} & 0.62 \\
\hline [SReq] & & & & no & mod-strg & positive & strong & $-[$ SReq] & 0.43 \\
\hline [Sche] & & & & & no & mod-strg & strong & & \\
\hline [Eqln] & & & & & & no & moderate & [Sche ] & 0.28 \\
\hline [all $S Q$ ] & & & & & & & no & [Eqln] & 0.14 \\
\hline \multicolumn{8}{|c|}{ Consistent judgements } & [all SQ] & 0.00 \\
\hline
\end{tabular}

Fig. 4. MACBETH matrix of qualitative judgments (left) and the corresponding validated weighting scale (right). (Note: "mod-strg", "strg-vstrg" and "vstrgextr" are abbreviations of "moderate or strong", "strong or very strong" and "very strong or extreme", respectively).

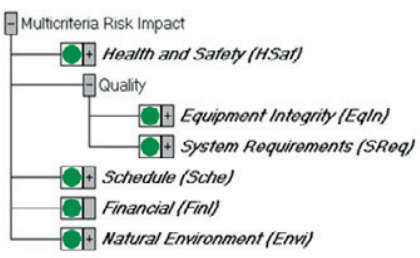

(a)

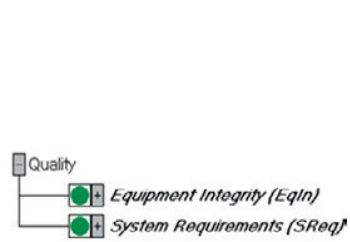

(b)

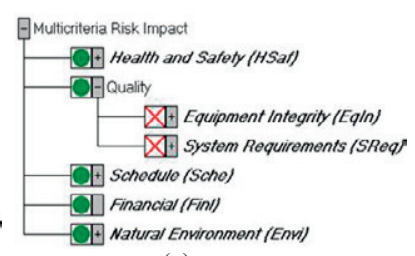

(c)

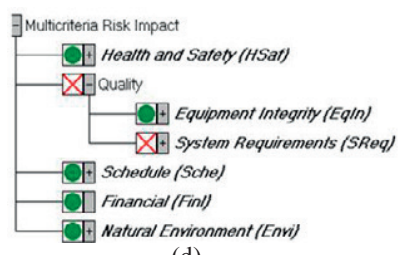

(d)

Fig. 5. Different possible ways for weighting criteria and area available in the recent hierarchical weighting component of the M-MACBETH DSS. 


\section{B. Subjective Probabilities}

Given the difficulties of organizations to elicit quantitative probabilities for risks when historical information is not available (or its use is not appropriate), RMs typically make use of qualitative scales (for example, low, medium, high) to probabilistically describe the likelihood of risk events. The use of probabilities in a discrete scale creates problems as it contributes for discontinuity jumps in the RM. MCDA methods can assist in building quantitative (subjective) probabilities using qualitative judgments from the DM following previous theoretical literature in the area using qualitative judgments to model probability [30] and using qualitative pairwise comparisons to model probabilities [3134], we will explore how MACBETH can be used to build a subjective probability scale. Naturally, MACBETH will be used in a different setting/logic in comparison to its common use in building multicriteria value models

\section{Risk Classification Procedure}

We propose exploring multicriteria classification procedures to classify risks from the VRM by severity. Building risk categories taking into account expected impacts and probabilities has been typically dealt in MCDA literature through the use of utilities (and lotteries) [19]. Given that the DM cannot choose between two risk events A and B, we propose developing alternative multicriteria procedures based on preference information over risk profiles to define the type of iso-risk lines that separate risk categories (i.e. isorisk thresholds that separate categories of risk are defined with the use of different colors of a traffic light system in a RM). In particular, procedures such as the ones used by Bana e Costa and Oliveira [35] will be further developed and adapted to the $\mathrm{RM}$ context.

\section{Value Risk-Matrix}

Using the methods proposed in A, B and C, a new RM can be designed, and we suggest creating a Value Risk Matrix (VRM). As an illustrative example, a preliminary design of VRM is shown in Fig. 6 (with multicriteria value scores in the $\mathrm{X}$-axis, probabilities in the $\mathrm{Y}$-axis, and risks classified into categories).

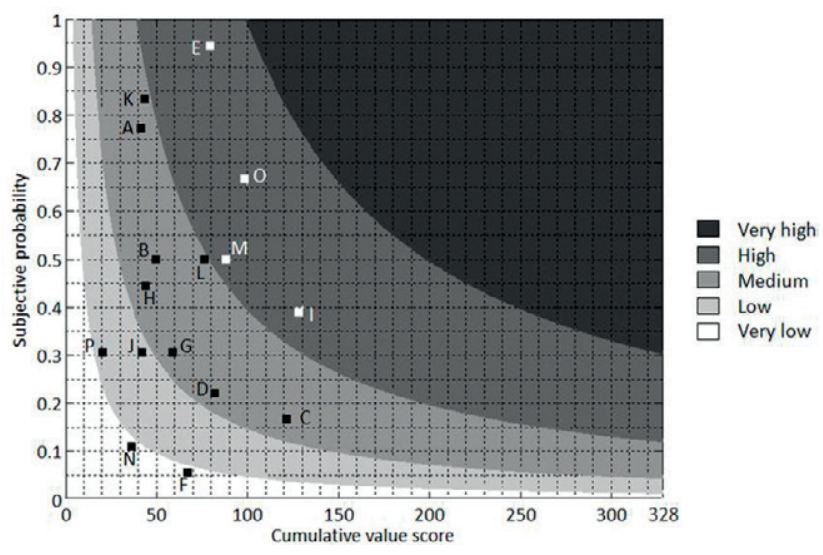

Fig. 6. Projecting the classification of risks into a VRM.

\section{E. Multicriteria Resource Allocation Model}

"Optimal" resource allocation demands for the quantitative information beyond the qualitative information that conventional RMs provide [7]. Combined with the VRM, the recent portfolio decision analysis component of the $\mathrm{M}$ MACBETH DSS can be built to assist the systematic analysis of the most effective set of interventions to reduce risk (i.e. which increase multicriteria impact value and/or decrease probabilities), while taking into account of cost and other constraints. Building multicriteria resource allocation models to assist the selection of interventions (and strategies) to reduce risks and that maximize value for money for the organization will help overcoming the problem of suboptimal allocations when using RMs.

It is worthwhile to note that the selection of the portfolio should be performed with the DM and the first step to find every efficient portfolio is to determine the added value (AV) of each mitigation action in relation to a baseline action. This AV can be calculated in two steps: a) first by calculating the adjusted value of a risk event (by multiplying the risk impact value by the probability of a risk event); and b) by measuring the difference between the value before and the adjusted value after adopting the mitigation action, which defined the AV of a mitigation action. A baseline mitigation action has null added value, i.e. if a mitigation action has negative added value, it should be regarded as globally unattractive and therefore excluded from the analysis. Then, it is necessary to determine the cost associated with each of the remaining mitigation action. Later, taking into account the available resources, the optimization approach, which solves the knapsack problem (for details see [36]). Fig. 7 shows all portfolios - i.e., combinations of mitigation actions - that can be formed with seven hypothetical mitigation actions and the efficient portfolio given a budget of $€ 39 \mathrm{~K}$. Note that the adopted Knapsack formulation covers other constraints than the mentioned budget constraints. These constraints can be translated as: inclusion and/or exclusion of certain actions in the portfolio; dependencies between actions; simultaneous integration of two actions; and exclusive integration of actions.

In practice, we propose using the M-MACBETH DSS since it allows, in an innovative way, to visualize the efficient frontier as well as take into account synergies and constraints between actions - a screen of the portfolio component of the M-MACBETH DSS is shown in Fig. 7.

\section{F. Modelling Uncertainty and Robustness Analysis}

Several types of uncertainty apply to the process of evaluating risks in VRMs, such as uncertainties regarding the measurement of risk impacts, regarding DM preferences and regarding the costs of risk mitigation actions (note that other types of uncertainties may also apply). When using the methods proposed in $\mathrm{A}$ to $\mathrm{D}$, the final question is how robust is the evaluation and classification of risks and of risk interventions. This requires the modeling of uncertainty sources and the availability of methods to carry out robustness analysis in an integrated form and to assist project risk management. 


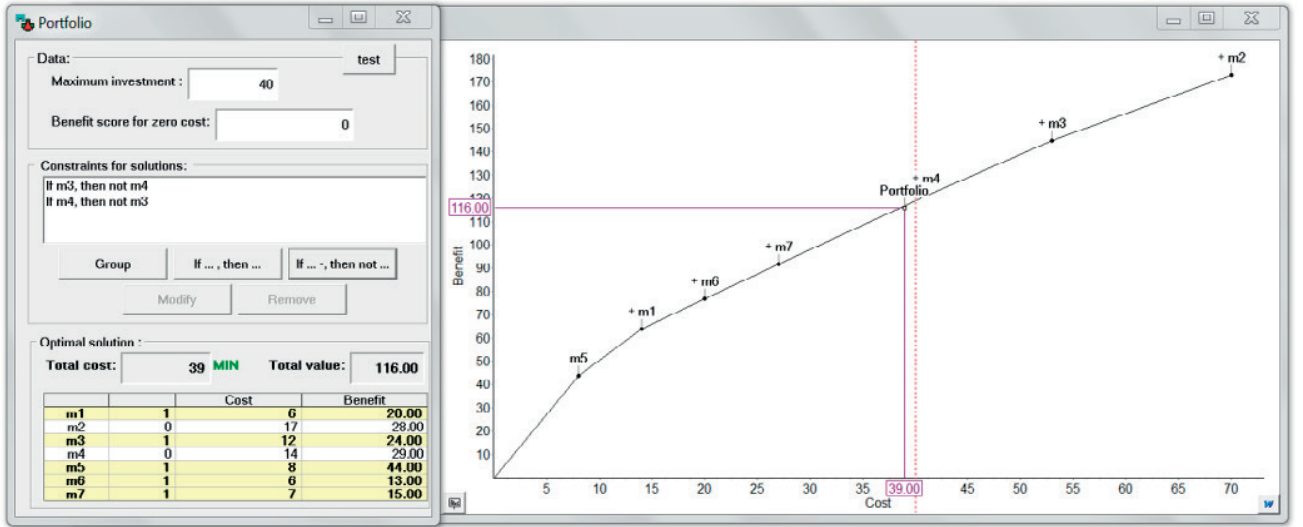

Fig. 7. Screen of the portfolio component of the M-MACBETH DSS, showing an optimization portfolio analysis of mitigation actions with several constraints.

Once the other methods within IRIS are developed and applied, uncertainty and robustness analyses will be built.

\section{CONCLUSIONS}

This study suggests methods and tools from multiple criteria and portfolio decision analysis tools, based on the MACBETH approach, to improve the design and the deployment of RMs in the context of prioritization of risks and of risk reductions. It shows how MACBETH method and software can be used within a social-technical process to build a VRM and to assist in the selection of mitigation actions. The social component includes the use of participatory methods to build preferences and a compromise and confidence between stakeholders who participate in risk management; and the technical component involves the modeling of the DM's preferences.

Within the IRIS project, the proposed methods are being developed and applied to several risk management case studies, including to the evaluation of health and safety risks and to the selection of risk mitigation actions in the Occupational Health and Safety Unit (OHSU) of the Regional Health Administration of Lisbon and Tagus Valley (RHA LVT). The aim is to build methods that are requisite (as defined in [37,38]) and can be used in risk management, as well as to build a new DSS to assist the use of VRM.

\section{ACKNOWLEDGMENT}

This work was funded by National Funds from the Portuguese Public Budget through FCT - Fundação para a Ciência e a Tecnologia, within the project PTDC/EGEGES/119230/2010.

\section{REFERENCES}

[1] T. Aven, Risk analysis: assessing uncertainties beyond expected values and probabilities, Wiley, 2008 ,

[2] D. Vose, Risk analysis: a quantitative guide, 3rd ed., Wiley, 2008,

[3] Project Management Institute, A guide to the project management body of knowledge: PMBOK guide, Project Management Institute, 2004,
[4] M.S.M. Figueiredo, M.D. Oliveira, Prioritizing risks based on multicriteria decision aid methodology: Development of methods applied to ALSTOM power, IEEE International Conference on Industrial Engineering and Engineering Management, Hong Kong, 2009, pp. $1568-1572$

[5] S.C. Ward, Assessing and managing important risks, International Journal of Project Management, 17, 1999, pp. 331-336.

[6] C. Alexander, Assessment of Operational Risk Capital, in: Risk Management: Challenge and Opportunity M. Frenkel, U. Hommel, M. Rudolf (Eds.)Springer, 2000, pp. 279-302.

[7] L.A. Cox Jr, Risk analysis of complex and uncertain systems, Springer, 2009 ,

[8] Quality and Patient Safety Directorate, Risk assessment tool and guidance (including guidance on application), Health Service Executive, 2011.

[9] H. Ni, Chen A., Chen N., Some extensions on risk matrix approach, Safety Science, 48, 2010, pp. 1269-1278.

[10] The MITRE Corporation, MITRE risk management toolkit, http:/www.mitre.org/work/sepo/toolkits/risk/ToolsTechniques/RiskMatr ix.html, 1999.

[11] SAP AG, Risk assessment with $\mathrm{SAP}{ }^{\circledR}$ EHS Management, SAP AG, 2012.

[12] L.A. Cox Jr., What's wrong with risk matrices?, Risk Analysis 28, 2008, pp. 497-512.

[13] P. Artzner, F. Delbaen, J.M. Eber, D. Heath, Coherent measures of risk, Mathematical Finance, 9, 1999, pp. 203-228.

[14] R.L. Dillon, E.M. Pate-Cornell, S.D. Guikema, Programmatic risk analysis for critical engineering systems under tight resource constraints, Operations Research, 51, 2003, pp. 354-370.

[15] Y.H. Kwak, F.T. Anbari, Analyzing project management research: Perspectives from top management journals, International Journal of Project Management, 27, 2009, pp. 435-446.

[16] A.S. Markowski, M.S. Mannan, Fuzzy risk matrix, Journal of Hazardous Materials, 159, 2008, pp. 152-157.

[17] R. Keeney, H. Raiffa, Decisions with multiple objectives: preferences and value tradeoffs, John Wiley \& Sons, 1976,

[18] D. Von Winterfeldt, W. Edwards, Decision analysis and behavioral research, Cambridge University Press, 1986,

[19] V. Belton, T.J. Stewart, Multiple criteria decision analysis: an integrated approach, Springer, 2002,

[20] C.A. Bana e Costa, J.-M. De Corte, V. J.-C., On the mathematical foundations of MACBETH, in: Multiple Criteria Decision Analysis: The State of the Art Surveys, 76, J. Figueira, Greco, S., Ehrgott, M. (Eds.), 2005, pp. 409-442. 
[21] C.A. Bana e Costa, J.C. Vansnick, MACBETH - An interactive path towards the construction of cardinal value functions, Internat Trans Oper Res 1, 1994, pp. 489-500.

[22] C.A. Bana e Costa, J.C. Vansnick, The MACBETH approach: Basic ideas, software and an application, in: Advances in Decision Analysis, N. Meskans, M. Roubens (Eds.),Dordrecht, Germany: Kluwer Academic Publishers, 1999, pp. 131-157.

[23] C.A. Bana e Costa, J.C. Lourenço, M.P. Chagas, J.C. Bana e Costa, Development of reusable bid evaluation models for the Portuguese Electric Transmission Company, Decision Analysis, 5, 2008, pp. 22-42.

[24] C.A. Bana e Costa, M.P. Chagas, A career choice problem: an example of how to use MACBETH to build a quantitative value model based on qualitative value judgments, European Journal of Operational Research, 103, 2004, pp. 323-331

[25] V. Clivillé, L. Berrah, G. Mauris, Quantitative expression and aggregation of performance measurements based on MACBETH multicriteria method, International Journal of Production Economics 105, 2007, pp. 171-189.

[26] F. Joerin, G. Cool, M.J. Rodriguez, M. Gignac, C. Bouchard, Using multi-criteria decision analysis to assess the vulnerability of drinking water utilities, Environmental Monitoring and Assessment 166, 2010, pp. 313-330.

[27] C.A. Bana e Costa, J.-M. De Corte, J.-C. Vansnick, MACBETH, International Journal of Information Technology and Decision Making, 11, 2012, pp. 359-387.

[28] R.L. Keeney, Value-focused thinking: a path to creative decisionmaking, Harvard University Press, 1992,

[29] C.A. Bana e Costa, L. Ensslin, E.C. Corrêa, J.-C. Vansnick, Decision Support Systems in action: Integrated application in a multicriteria decision aid process, European Journal of Operational Research, 113, 1999, pp. 315-335.

[30] K.-P. Schott, A model to support the assessment of subjective probabilities, Theory and Decision 12, 1980, pp. 173-183.

[31] S. Monti, G. Carenini, Dealing with the expert inconsistency in probability elicitation, IEEE Transactions on Knowledge and Data Engineering, 12, 2000, pp. 499-508.

[32] J.L. Riggs, S.B. Brown, R.P. Trueblood, Integration of technical, cost, and schedule risks in project management, Computers and Operations Research, 21, 1994, pp. 521-533.

[33] T.L. Saaty, Risk-its priority and probability: the analytic hierarchy process, Risk Analysis, 7, 1987, pp. 159-172.

[34] P. Szwed, J. Rene van Dorp, J.R.W. Merrick, T.A. Mazzuchi, A. Singh, A Bayesian paired comparison approach for relative accident probability assessment with covariate information, European Journal of Operational Research, 169, 2006, pp. 157-177.

[35] C.A. Bana e Costa, R.C. Oliveira, Assigning priorities for maintenance, repair and refurbishment in managing a municipal housing stock, European Journal of Operational Research, 138 2002, pp. 380-391.

[36] J.C. Lourenço, A. Morton, C.A. Bana e Costa, PROBE - a multicriteria decision support system for portfolio robustness evaluation, Decision Support Systems, 54, 2012, pp. 534-550.

[37] L.D. Phillips, A theory of requisite decision models, Acta Psychologica, 56, 1984, pp. 29-48.

[38] L.D. Phillips, C.A. Bana e Costa, Transparent prioritisation, budgeting and resource allocation with multi-criteria decision analysis and decision conferencing, Annals of Operations Research 154, 2007, pp. 51-68. 